\title{
EXTREMAL PROPERTIES FOR DISSECTIONS OF CONVEX 3-POLYTOPES*
}

\author{
JESÚS A. DE LOERA ${ }^{\dagger}$, FRANCISCO SANTOS ${ }^{\ddagger}$, AND FUMIHIKO TAKEUCHI ${ }^{\S}$
}

\begin{abstract}
A dissection of a convex $d$-polytope is a partition of the polytope into $d$-simplices whose vertices are among the vertices of the polytope. Triangulations are dissections that have the additional property that the set of all its simplices forms a simplicial complex. The size of a dissection is the number of $d$-simplices it contains. This paper compares triangulations of maximal size with dissections of maximal size. We also exhibit lower and upper bounds for the size of dissections of a 3-polytope and analyze extremal size triangulations for specific nonsimplicial polytopes: prisms, antiprisms, Archimedean solids, and combinatorial $d$-cubes.
\end{abstract}

Key words. dissection, triangulation, mismatched region, lattice polytope, combinatorial $d$ cube, prism, antiprism, Archimedean solid

AMS subject classifications. 52B45, 52B05, 52B70, 52B55

PII. S0895480199366238

1. Introduction. Let $\mathcal{A}$ be a point configuration in $\boldsymbol{R}^{d}$ with its convex hull $\operatorname{conv}(\mathcal{A})$ having dimension $d$. A set of $d$-simplices with vertices in $\mathcal{A}$ is a dissection of $\mathcal{A}$ if no pair of simplices has an interior point in common and their union equals $\operatorname{conv}(\mathcal{A})$. A dissection is a triangulation of $\mathcal{A}$ if in addition any pair of simplices intersects at a common face (possibly empty). The size of a dissection is the number of $d$-simplices it contains. We say that a dissection is mismatching when it is not a triangulation (i.e., it is not a simplicial complex). In this paper we study mismatching dissections of maximal possible size for a convex polytope and compare them with maximal triangulations. This investigation is related to the study of Hilbert bases and the hierarchy of covering properties for polyhedral cones which is relevant in algebraic geometry and integer programming (see [5, 10, 24]). Maximal dissections are relevant also in the enumeration of interior lattice points and its applications (see $[2,15]$ and references therein).

It was first shown by Lagarias and Ziegler that dissections of maximal size turn out to be, in general, larger than maximal triangulations, but their example uses interior points [16]. Similar investigations were undertaken for mismatching minimal dissections and minimal triangulations of convex polytopes [4]. In this paper we augment previous results by showing that it is possible to have simultaneously, in the same 3-polytope, that the size of a mismatching minimal (maximal) dissection is smaller (larger) than any minimal (maximal) triangulation. In addition, we show that the gap between the size of a mismatching maximal dissection and a maximal triangulation can grow linearly on the number of vertices and that this occurs already for a family of simplicial convex 3-polytopes. A natural question is how different

* Received by the editors December 22, 1999; accepted for publication (in revised form) December 12, 2000; published electronically February 23, 2001.

http://www.siam.org/journals/sidma/14-2/36623.html

${ }^{\dagger}$ Department of Mathematics, University of California, Davis, CA 95616 (deloera@math. ucdavis.edu). The research of this author was partially supported by NSF grant DMS-0073815.

${ }^{\ddagger}$ Departmento de Matemáticas, Estadistica y Comput., Universidat de Cantabria, E-39005 Santander, Cantabria, Spain (santos@matesco.unican.es). The research of this author was partially supported by grant PB97-0358 of the Spanish Dirección General de Investigación Científica y Técnica.

${ }_{\S}$ Department of Information Science, University of Tokyo, Tokyo, 113-0033, Japan (fumi@is.s. u-tokyo.ac.jp). 
are the upper and lower bounds for the size of mismatching dissections versus those bounds known for triangulations (see [21]). We prove lower and upper bounds on their size with respect to the number of vertices for dimension three and exhibit examples showing that our technique of proof fails already in dimension four. Here is the first summary of results.

THEOREM 1.1.

(1) There exists an infinite family of convex simplicial 3-polytopes with increasing number of vertices whose mismatching maximal dissections are larger than their maximal triangulations. This gap is linear in the number of vertices (Corollary 2.2).

(2) (a) There exists a lattice 3-polytope with eight vertices containing no other lattice point other than its vertices whose maximal dissection is larger than its maximal triangulations.

(b) There exists a 3-polytope with eight vertices for which, simultaneously, its minimal dissection is smaller than minimal triangulations and maximal dissection is larger than maximal triangulations (Proposition 2.3).

(3) If $D$ is a mismatching dissection of a 3-polytope with $n$ vertices, then the size of $D$ is at least $n-2$. In addition, the size of $D$ is bounded above by $\left(\begin{array}{c}n-2 \\ 2\end{array}\right)$ (Proposition 3.2).

A consequence of our third point is that the result of [4], stating a linear gap between the size of minimal dissections and minimal triangulations, is best possible. The results are discussed in sections 2 and 3 .

The last section presents a study of maximal and minimal triangulations for combinatorial $d$-cubes, three-dimensional prisms and antiprisms, as well as other Archimedean polytopes. The following theorem and table summarize the main results.

THEOREM 1.2.

(1) There is a constant $c>1$ such that for every $d \geq 3$ the maximal triangulation among all possible combinatorial d-cubes has size at least $c^{d} d$ ! (Proposition 4.1).

(2) For a three-dimensional m-prism, in any of its possible coordinatizations, the size of a minimal triangulation is $2 m-5+\left\lceil\frac{m}{2}\right\rceil$. For an m-antiprism, in any of its possible coordinatizations, the size of a minimal triangulation is $3 m-5$ (Proposition 4.3). The size of a maximal triangulation of an $m$-prism depends on the coordinatization, and in certain natural cases it is $\left(m^{2}+m-6\right) / 2$ (Proposition 4.4).

(3) Table 1 specifies sizes of the minimal and maximal triangulations for some Platonic and Archimidean solids. These results were obtained via integer programming calculations using the approach described in [8]. All computations used the canonical symmetric coordinatizations for these polytopes [6]. The number of vertices is indicated in parenthesis (Remark 4.5).

2. Maximal dissections of 3-polytopes. We introduce some important definitions and conventions: We denote by $Q_{m}$ a convex $m$-gon with $m$ an even positive integer. Let $v_{1} v_{2}$ and $u_{1} u_{2}$ be two edges parallel to $Q_{m}$, orthogonal to each other, on opposite sides of the plane containing $Q_{m}$, and such that the four segments $v_{i} u_{j}$ intersect the interior of $Q_{m}$. We suppose that $v_{1} v_{2}$ and $u_{1} u_{2}$ are not parallel to any diagonal or edge of $Q_{m}$. The convex hull $P_{m}$ of these points has $m+4$ vertices and it is a simplicial polytope. We will call the north (respectively, south) vertex of $Q_{m}$ the one which maximizes (respectively, minimizes) the scalar product with the vector 
TABLE 1

Sizes of extremal triangulations of Platonic and Archimidean solids.

\begin{tabular}{|c|c|c|}
\hline$P$ & $\left|T_{\min }(P)\right|$ & $\left|T_{\max }(P)\right|$ \\
\hline Icosahedron (12) & 15 & 20 \\
Dodecahedron (20) & 23 & 36 \\
Cuboctahedron (12) & 13 & 17 \\
Icosidodecahedron (30) & 45 & $?$ \\
Truncated Tetrahedron (12) & 10 & 13 \\
Truncated Octahedron (24) & 27 & $?$ \\
Truncated Cube (24) & 25 & 48 \\
Small Rhombicuboctahedron (24) & 35 & $?$ \\
Pentakis Dodecahedron (32) & 54 & $?$ \\
Rhombododecahedron (14) & 12 & 21 \\
\hline
\end{tabular}

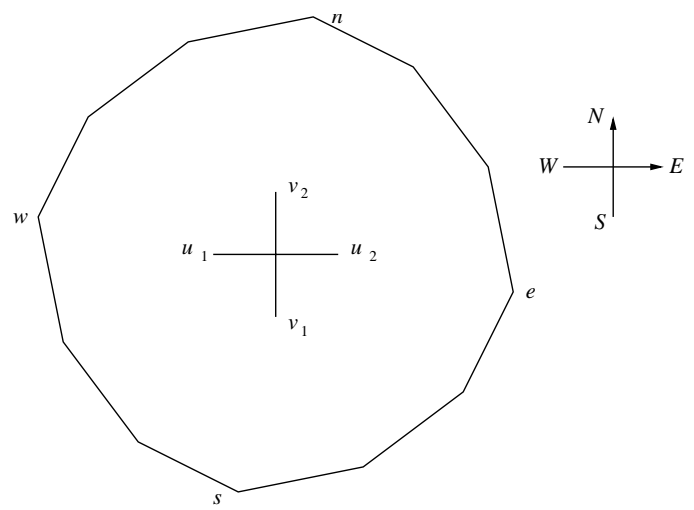

FIG. 1. North, south, east, and west vertices.

$v_{2}-v_{1}$. Similarly, we will call east (west) the vertex which maximizes (minimizes) the scalar product with $u_{2}-u_{1}$. We denote these four vertices $n, s, e$ and $w$, respectively. See Figure 1.

We say that a directed path of edges inside $Q_{m}$ is monotone in the direction $v_{1} v_{2}$ (respectively, $u_{1} u_{2}$ ) when the vertices of the path appear in the path following the same order given by the scalar product with $v_{2}-v_{1}$ (respectively, $u_{2}-u_{1}$ ). An equivalent formulation is that any line orthogonal to $v_{1} v_{2}$ cuts the path in at most one point. We remark that by our choice of $v_{1} v_{2}$ and $u_{1} u_{2}$ all vertices of $Q_{m}$ are ordered by the values of their scalar products with $v_{2}-v_{1}$ and also with respect to $u_{2}-u_{1}$. In the same way, a sequence of vertices of $Q_{m}$ is ordered in the direction of $v_{1} v_{2}$ (respectively, $u_{1} u_{2}$ ) if the order is the same as the one provided by using the values of the scalar products of the points with the vector $v_{2}-v_{1}$ (respectively, $u_{2}-u_{1}$ ). Consider the two orderings induced by the directions of $v_{1} v_{2}$ and $u_{1} u_{2}$ on the set of vertices of $Q_{m}$. Let us call horizontal (respectively, vertical) any edge joining two consecutive vertices in the direction of $v_{1} v_{2}$ (respectively, of $u_{1} u_{2}$ ). As an example, if $Q_{m}$ is regular, then the vertical edges in $Q_{m}$ form a zig-zag path as shown in Figure 2 .

Our examples in this section will be based on the following observation and are inspired by a similar analysis of maximal dissections of dilated empty lattice tetrahedra in $\boldsymbol{R}^{3}$ by Lagarias and Ziegler [16]: Let $R_{m}$ be the convex hull of the $m+2$ vertices consisting of the $m$-gon $Q_{m}$ and $v_{1}, v_{2} . R_{m}$ is exactly one half of the polytope $P_{m}$. Consider a triangulation $T_{0}$ of $Q_{m}$ and a path $\Gamma$ of edges of $T_{0}$ monotone with 

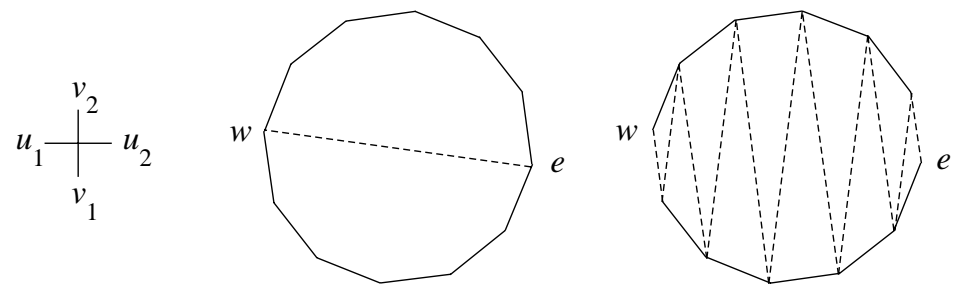

FIG. 2. The minimal monotone path (middle) and the maximal monotone path made by the vertical edges (right) in the direction $u_{1} u_{2}$.

respect to the direction $u_{1} u_{2}$. Observe that $\Gamma$ divides $T_{0}$ in two regions, which we will call the "north" and the "south." Then, the following three families of tetrahedra form a triangulation $T$ of $R_{m}$ : the edges of $\Gamma$ joined to the edge $v_{1} v_{2}$, the southern triangles of $T_{0}$ joined to $v_{1}$, and the northern triangles of $T_{0}$ joined to $v_{2}$ (see Figure 3 ). Moreover, all the triangulations of $R_{m}$ are obtained in this way: Any triangulation $T$ of $R_{m}$ induces a triangulation $T_{0}$ of $Q_{m}$. The link of $v_{1} v_{2}$ in $T$ is a monotone path of edges contained in $T_{0}$ and it divides $T_{0}$ in two regions joined, respectively, to $v_{1}$ and $v_{2}$.
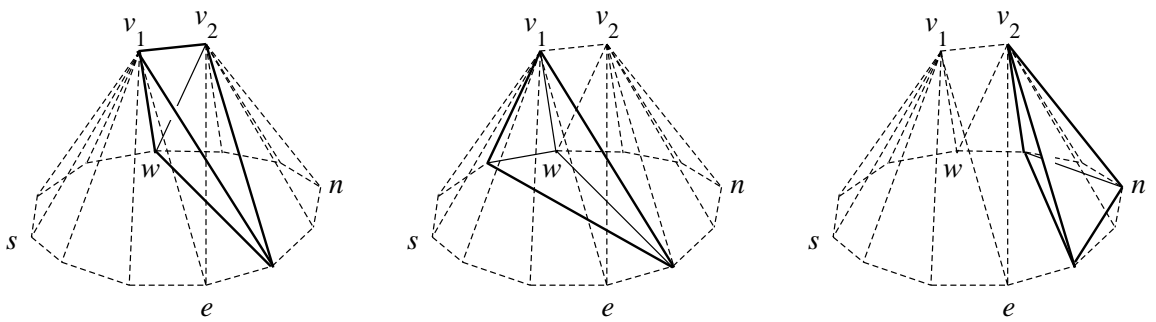

FIG. 3. Three types of tetrahedra in $R_{m}$.

Using the Cayley trick, one can also think of the triangulations of $R_{m}$ as the fine mixed subdivisions of the Minkowski sum $Q_{m}+v_{1} v_{2}$ (see [13] and references therein).

The size of a triangulation of $R_{m}$ equals $m-2+|\Gamma|$, where $|\Gamma|$ is the number of edges in the path $\Gamma$. There is a unique minimal path in $Q_{m}$ of length one (Figure 2 , middle) and a unique maximal path of length $m-1$ (Figure 2, right). Hence the minimal and maximal triangulations of $R_{m}$ have, respectively, $m-1$ and $2 m-3$ tetrahedra. The maximal triangulation is unique, but the minimal one is not: after choosing the diagonal in $\Gamma$ the rest of the polygon $Q_{m}$ can be triangulated in many ways. From the above discussion regarding $R_{m}$ we see that we could independently triangulate each of the two halves of $P_{m}$ with any number of tetrahedra from $m-1$ to $2 m-3$. Hence $P_{m}$ has dissections of sizes going from $2 m-2$ to $4 m-6$. Among the triangulations of $P_{m}$, we will call halving triangulations those that triangulate the two halves of $P_{m}$. Equivalently, the halving triangulations are those which do not contain any of the four edges $v_{i} u_{j}$.

Proposition 2.1. Let $P_{m}$ be as described above with $Q_{m}$ being a regular m-gon. No triangulation of $P_{m}$ has more than $\frac{7 m}{2}+1$ tetrahedra. On the other hand, there are mismatching dissections of $P_{m}$ with $4 m-6$ tetrahedra.

Proof. Let $T$ be a triangulation of $P_{m}$. It is an easy application of Euler's formulas for the 3 -ball and 2-sphere that the number of tetrahedra in a triangulation of any 
3-ball without interior vertices equals the number of vertices plus interior edges minus three (such a formula appears for instance in [9]). Hence our task is to prove that $T$ has at most $\frac{5 m}{2}$ interior edges. For this, we classify the interior edges according to how many vertices of $Q_{m}$ they are incident to. There are only four edges not incident to any vertex of $Q_{m}$ (the edges $v_{i} u_{j}, i, j \in\{1,2\}$ ). Moreover, $T$ contains at most $m-3$ edges incident to two vertices of $Q_{m}$ (i.e., diagonals of $Q_{m}$ ), since in any family of more than $m-3$ such edges there are pairs which cross each other. Thus, it suffices to prove that $T$ contains at most $\frac{3 m}{2}-1$ edges incident to just one vertex of $Q_{m}$, i.e., of the form $v_{i} p$ or $u_{i} p$ with $p \in Q_{m}$.

Let $p$ be any vertex of $Q_{m}$. If $p$ equals $w$ or $e$, then the edges $p v_{1}$ and $p v_{2}$ are both in the boundary of $P_{m}$; for any other $p$, exactly one of $p v_{1}$ and $p v_{2}$ is on the boundary and the other one is interior. Moreover, we claim that if $p v_{i}$ is an interior edge in a triangulation $T$, then the triangle $p v_{1} v_{2}$ appears in $T$. This is so because there is a plane containing $p v_{i}$ and having $v_{3-i}$ as the unique vertex on one side. At the same time the link of $p v_{i}$ is a cycle going around the edge. Hence $v_{3-i}$ must appear in the link of $p v_{i}$. It follows from the above claim that the number of interior edges of the form $p v_{i}$ in $T$ equals the number of vertices of $Q_{m}$ other than $w$ and $e$ in the link of $v_{1} v_{2}$. In a similar way, the number of interior edges of the form $p u_{i}$ in $T$ equals the number of vertices of $Q_{m}$ other than $n$ and $s$ in the link of $u_{1} u_{2}$. In other words, if we call $\Gamma_{u}=\operatorname{link}_{T}\left(v_{1} v_{2}\right) \cap Q_{m}$ and $\Gamma_{v}=\operatorname{link}_{T}\left(u_{1} u_{2}\right) \cap Q_{m}$ (the $u, v$ in the index and of the vertices are reversed because in this way $\Gamma_{u}$ is monotone with respect to $u_{1} u_{2}$, and $\Gamma_{v}$ with respect to $v_{1} v_{2}$ ), then the number of interior edges in $T$ incident to exactly one vertex of $Q_{m}$ equals $\mid$ vertices $\left(\Gamma_{v}\right)|+|$ vertices $\left(\Gamma_{u}\right) \mid-4$. Our goal is to bound this number. As an example, Figure 4 shows the intersection of $Q_{m}$ with a certain triangulation of $P_{m}(m=12)$. The link of $v_{1} v_{2}$ in this triangulation is the chain of vertices and edges $w a b u_{1} n u_{2} c e$ (the star of $v_{1} v_{2}$ is marked in thick and grey in the figure). $\Gamma_{u}$ consists of the chains $w a b$ and $c e$ and the isolated vertex $n$. In turn, the link of $u_{1} u_{2}$ is the chain $n v_{1} s$ and $\Gamma_{v}$ consists of the isolated vertices $n$ and $s$.

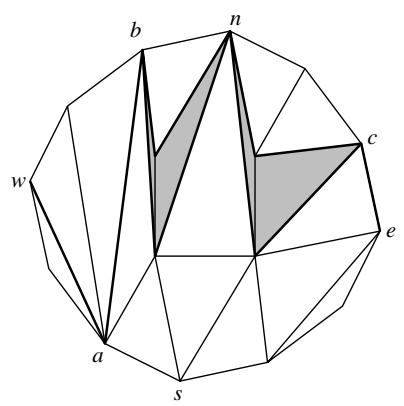

FIG. 4. Illustration of the proof of Proposition 2.1.

Observe that $\Gamma_{v}$ has at most three connected components because it is obtained by removing from $\operatorname{link}_{T}\left(u_{1} u_{2}\right)$ (a path) the parts of it incident to $v_{1}$ and $v_{2}$, if any. Each component is monotone in the direction of $v_{1} v_{2}$ and the projections of any two components to a line parallel to $v_{1} v_{2}$ do not overlap. The sequence of vertices of $Q_{m}$ ordered in the direction of $v_{1} v_{2}$ can have a pair of consecutive vertices contained in $\Gamma_{v}$ only where there is a horizontal edge in $\Gamma_{v}$ or in the (at most) two discontinuities of $\Gamma_{v}$. This is true because $Q_{m}$ is a regular $m$-gon.

We denote $n_{h o r}$ the number of horizontal edges in $\Gamma_{v}$ and $n_{h o r}^{\prime}$ this number plus the number of discontinuities in $\Gamma_{v}$ (hence $n_{\text {hor }}^{\prime} \leq n_{\text {hor }}+2$ ). Every nonhorizontal 
edge of $\Gamma_{v}$ produces a jump of at least two in the $v_{1} v_{2}$-ordering of the vertices of $P_{m}$; hence we have

$$
\left|\operatorname{vertices}\left(\Gamma_{v}\right)\right|-1-n_{h o r}^{\prime} \leq \frac{m-1-n_{h o r}^{\prime}}{2} .
$$

Analogously, and with the obvious similar meaning for $n_{\text {vert }}$ and $n_{\text {vert }}^{\prime}$,

$$
\left|\operatorname{vertices}\left(\Gamma_{u}\right)\right|-1-n_{\text {vert }}^{\prime} \leq \frac{m-1-n_{\text {vert }}^{\prime}}{2} .
$$

Since $\Gamma_{u} \cup \Gamma_{v}$ can be completed to a triangulation of $Q_{m}$, and exactly four noninterior edges of $Q_{m}$ are horizontal or vertical, we have $n_{\text {hor }}+n_{\text {vert }} \leq(m-3)+4=m+1$, i.e., $n_{\text {hor }}^{\prime}+n_{\text {vert }}^{\prime} \leq m+5$. Hence

$$
\left|\operatorname{vertices}\left(\Gamma_{v}\right)\right|+\left|\operatorname{vertices}\left(\Gamma_{u}\right)\right| \leq\left\lfloor\frac{2 m+2+n_{\text {hor }}^{\prime}+n_{\text {vert }}^{\prime}}{2}\right\rfloor \leq\left\lfloor\frac{3 m+7}{2}\right\rfloor=\frac{3 m}{2}+3 .
$$

Thus, there are at most $\frac{3 m}{2}-1$ interior edges in $T$ of the form $p v_{i}$ or $p u_{i}$ and at most $\frac{5 m}{2}$ interior edges in total, as desired.

Corollary 2.2. The polytope $P_{m}$ described above has the following properties:

- It is a simplicial 3-polytope with $m+4$ vertices.

- Its maximal dissection has at least $4 m-6$ tetrahedra.

- Its maximal triangulation has at most $\frac{7 m}{2}+1$ tetrahedra.

In particular, the gap between sizes of the maximal dissection and maximal triangulation is linear on the number of vertices.

Three remarks are in order: First, the size of the maximal triangulation for $P_{m}$ may depend on the coordinates or, more specifically, on which diagonals of $Q_{m}$ intersect the tetrahedron $v_{1} v_{2} u_{1} u_{2}$. Second, concerning the size of the minimal triangulation of $P_{m}$, we can easily describe a triangulation of $P_{m}$ with only $m+5$ tetrahedra: let the vertices $n, s, e$, and $w$ be as defined above (see Figure 1) and let us call northeast, northwest, southeast, and southwest the edges in the arcs ne, nw, se, and $s w$ in the boundary of $Q_{m}$. Then, the triangulation consists of the five tetrahedra $v_{1} v_{2} u_{1} u_{2}, v_{1} v_{2} u_{1} w, v_{1} v_{2} u_{2} e, v_{1} u_{1} u_{2} s$, and $v_{2} u_{1} u_{2} n$ (shown in the left part of Figure $5)$ together with the edges $v_{2} u_{2}, v_{2} u_{1}, v_{1} u_{2}$, and $v_{1} u_{1}$ joined, respectively, to the northeast, northwest, southeast, and southwest edges of $Q_{m}$. The right part of Figure 5 shows the result of slicing through the triangulation by the plane containing the polygon $Q_{m}$.

Finally, although the corollary above states a difference between maximal dissections and maximal triangulations only for $P_{m}$ with $m>14$, experimentally we have observed there is a gap already for $m=8$. Now we discuss two other interesting examples. The following proposition constitutes the proof of Theorem 1.1 (2).

PROPOSITION 2.3.

(1) Consider the following eight points in $\boldsymbol{R}^{3}$ :

- The vertices $s=(0,0,0), e=(1,0,0), w=(0,1,0)$, and $n=(1,1,0)$ of a square in the plane $z=0$.

- The vertices $v_{1}=(-1,0,1)$ and $v_{2}=(1,1,1)$ of a horizontal edge above the square, and

- the vertices $u_{1}=(0,1,-1)$ and $u_{2}=(2,0,-1)$ of a horizontal edge below the square.

These eight points are the vertices of a polytope $P$ whose only integer points are precisely its eight vertices and with the following properties: 

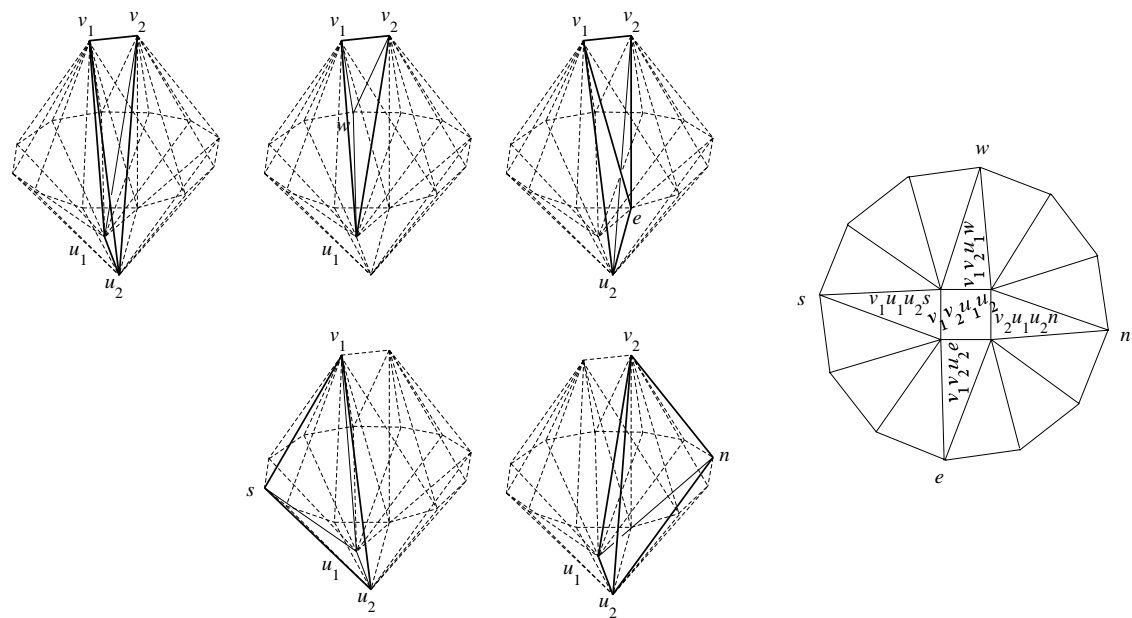

Fig. 5. For the triangulation of $P_{m}$ with $m+5$ tetrahedra, its five central tetrahedra (left) and the intersection of the triangulation with the polygon $Q_{m}$ (right) are shown. The four interior vertices are the intersection points of the edges $v_{1} u_{1}, v_{1} u_{2}, v_{2} u_{1}$, and $v_{2} u_{2}$ with the plane containing $Q_{m}$.

(a) Its (unique) maximal dissection has 12 tetrahedra. All of them are unimodular, i.e., they have volume $1 / 6$.

(b) Its (several) maximal triangulations have 11 tetrahedra.

(2) For the 3-polytope with vertices $u_{1}=(1,0,0), w=(1,0,1), v_{1}=(-1,0,0)$, $s=(-1,0,-1), v_{2}=(0,1,1), n=(1,1,1), u_{2}=(0,1,-1), e=(-1,1,-1)$, the sizes of its (unique) minimal dissection and (several) minimal triangulations are 6 and 7, respectively, and the sizes of its (several) maximal triangulations and (unique) maximal dissection are 9 and 10, respectively.

Proof. The polytopes constructed are quite similar to $P_{4}$ constructed earlier except that $Q_{4}$ is nonregular (in part (2)) and the segments $u_{1} u_{2}$ and $v_{1} v_{2}$ are longer and are not orthogonal, thus ending with different polytopes. The polytopes are shown in Figure 6. Figure 7 describes a maximal dissection of each of them in five parallel slices. Observe that both polytopes have four vertices in the plane $y=0$ and another four in the plane $y=1$. Hence the first and last slices in parts (a) and (b) of Figure 7 completely describe the polytope.
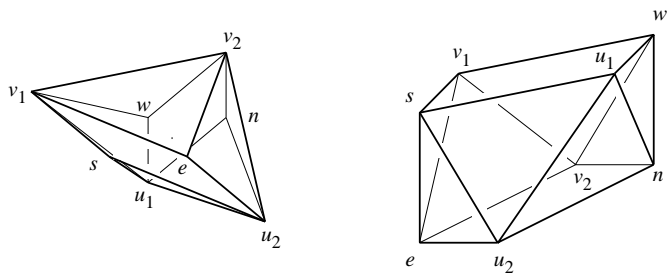

FIG. 6. The two polytopes in Proposition 2.3.

(1) The vertices in the planes $y=0$ and $y=1$ form convex quadrangles whose only integer points are the four vertices. This proves that the eight points are in convex position and that the polytope $P$ contains no integer point other than its vertices. Let us now prove the assertions on maximal dissections and triangulations 


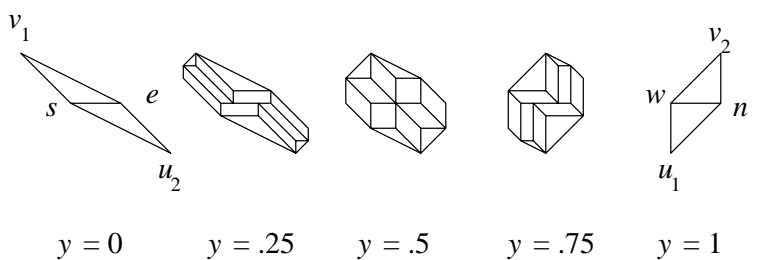

(a)
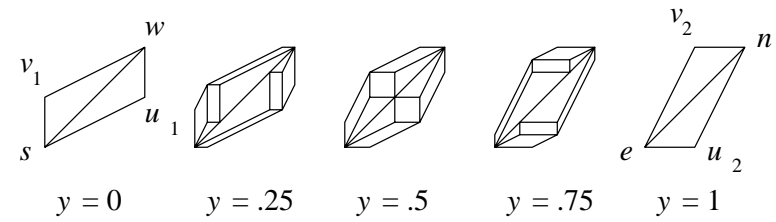

(b)

FIG. 7. Five 2-dimensional slices of the maximal dissections of the polytopes in Proposition 2.3. The first and last slices are two facets of the polytopes containing all the vertices.

of $P$ :

(a) Consider the paths of length three $\Gamma_{v}=\{e s n w\}$ and $\Gamma_{u}=\{$ sewn $\}$, which are monotone, respectively, in the directions orthogonal to $v_{1} v_{2}$ and $u_{1} u_{2}$. Using them, we can construct two triangulations of size five of the polytopes conv $\left(n s e w v_{1} v_{2}\right)$ and $\operatorname{conv}\left(\right.$ nsewu $\left._{1} u_{2}\right)$, respectively. However, they do not fill $P$ completely. There is space left for the tetrahedra $s w v_{1} u_{1}$ and $e n v_{2} u_{2}$. This gives a dissection of $P$ with 12 tetrahedra. All the tetrahedra are unimodular, so no bigger dissection is possible.

(b) A triangulation of size 11 can be obtained using the same idea as above, but with paths $\Gamma_{v}$ and $\Gamma_{u}$ of lengths three and two, respectively, which can be taken from the same triangulation of the square nswe.

To prove that no triangulation has bigger size, it suffices to show that $P$ does not have any unimodular triangulation. This means all tetrahedra have volume $1 / 6$. We start by recalling a well-known fact (see Corollary 4.5 in [25]). A lattice tetrahedron has volume $1 / 6$ if and only if each of its vertices $v$ lies in a consecutive lattice plane parallel to the supporting plane of the opposite facet to $v$. Two parallel planes are said to be consecutive if their equations are $a x+b y+c z=d$ and $a x+b y+c z=d-1$.

Suppose that $T$ is a unimodular triangulation of $P$. We will first prove that the triangle $u_{1} u_{2} e$ is in $T$. The triangular facet $u_{1} u_{2} s$ of $P$, lying in the hyperplane $x+2 y+2 z=0$, has to be joined to a vertex in the plane $x+2 y+2 z=1$. The two possibilities are $e$ and $v_{1}$. With the same argument, if the tetrahedron $u_{1} u_{2} s v_{1}$ is in $T$, its facet $u_{1} u_{2} v_{1}$, which lies in the hyperplane $2 x+4 y+3 z=1$, will be joined to a vertex in $2 x+4 y+3 z=2$, and the only one is $e$. This finishes the proof that $u_{1} u_{2} e$ is a triangle in $T$. Now $u_{1} u_{2} e$ is in the plane $x+2 y+z=1$ and must be joined to a vertex in $x+2 y+z=2$, i.e., to $w$. Hence $u_{1} u_{2} e w$ is in $T$ and, in particular, $T$ uses the edge ew. $P$ is symmetric under the rotation of order two on the axis $\left\{z=0, x=\frac{1}{2}\right\}$. Applying this symmetry to the previous arguments we conclude that $T$ uses the edge $n s$ too. However, this is impossible since the edges $n s$ and $e w$ cross each other.

(2) This polytope almost fits the description of $P_{4}$, except for the fact that the edges $v_{1} u_{1}, v_{2} u_{2}$ intersect the boundary and not the interior of the planar quadrangle 
nsew. With the general techniques we have described, it is easy to construct halving dissections of this polytope with sizes from 6 to 10. Combinatorially, the polytope is a 4 -antiprism. Hence, Proposition 4.3 shows that its minimal triangulation has seven tetrahedra. The rest of the assertions in the statement were proved using the integer programming approach proposed in [8], which we describe in Remark 4.5. We have also verified them by enumerating all triangulations $[19,29]$. It is interesting to observe that if we perturb the coordinates a little so that the planar quadrilateral $u_{1} v_{1} u_{2} e$ becomes a tetrahedron with the right orientation and without changing the face lattice of the polytope, then the following becomes a triangulation with 10 tetrahedra: $\left\{u_{1} u_{2} s e, u_{1} u_{2} e v_{1}, u_{1} u_{2} v_{1} w, u_{1} u_{2} w n, v_{1} v_{2} e n, v_{1} v_{2} n w, u_{1} v_{1} s e\right.$, $\left.v_{1} u_{2} e w, u_{2} w n e, v_{1} w n e\right\}$.

3. Bounds for the size of a dissection. Let $D$ be a dissection of a $d$-polytope $P$. Say two $(d-1)$-simplices $S_{1}$ and $S_{2}$ of $D$ intersect improperly in a $(d-1)$ hyperplane $H$ if both lie in $H$, are not identical, and they intersect with a nonempty relative interior. Consider the following auxiliary graph: take as nodes the $(d-1)$ simplices of a dissection, and say that two $(d-1)$-simplices are adjacent if they intersect improperly in a certain hyperplane. A mismatched region is the subset of $\boldsymbol{R}^{d}$ that is the union of $(d-1)$-simplices over a connected component of size larger than one in such a graph. Later, in Proposition 3.4, we will show some of the complications that can occur in higher dimensions.

Define the simplicial complex of a dissection as all the simplices of the dissection together with their faces, where only faces that are identical (in $\boldsymbol{R}^{d}$ ) are identified. This construction corresponds intuitively to an inflation of the dissection, where for each mismatched region we move the two groups of $(d-1)$-simplices slightly apart leaving the relative boundary of the mismatched region joined. Clearly, the simplicial complex of a dissection may be not homeomorphic to a ball.

The deformed $d$-simplices intersect properly, and the mismatched regions become holes. The numbers of vertices and $d$-simplices do not change.

Lemma 3.1. All mismatched regions for a dissection of a convex 3-polytope $P$ are convex polygons with all vertices among the vertices of $P$. Distinct mismatched regions have disjoint relative interiors.

Proof. Let $Q$ be a mismatched region and $H$ the plane containing it. Since a mismatched region is a union of overlapping triangles, it is a polygon in $H$ with a connected interior. If two triangles forming the mismatched region have interior points in common, they should be facets of tetrahedra in different sides of $H$. Otherwise the two tetrahedra would have interior points in common, contradicting the definition of dissection. Triangles which are facets of tetrahedra in one side of $H$ cover $Q$. Triangles coming from the other side of $H$ also cover $Q$.

Now take triangles coming from one side. As mentioned above, they have no interior points in common. Their vertices are among the vertices of the tetrahedra in the dissection, thus among the vertices of the polytope $P$. Hence the vertices of the triangles are in convex position, thus the triangles are forming a triangulation of a convex polygon in $H$ whose vertices are among the vertices of $P$.

For the second claim, suppose there were distinct mismatched regions having an interior point in common. Then their intersection should be an interior segment for each. Let $Q$ be one of the mismatched regions. It is triangulated in two different ways each coming from the tetrahedra in one side of the hyperplane. The triangles in either triangulation cannot intersect improperly with the interior segment. Thus the two triangulations of $Q$ have an interior diagonal edge in common. This means the 
triangles in $Q$ consist of more than one connected component of the auxiliary graph, contradicting the definition of mismatched region.

Proposition 3.2.

(1) The size of a mismatching dissection $D$ of a convex 3-polytope with $n$ vertices is at least $n-2$.

(2) The size of a dissection of a 3-polytope with $n$ vertices is bounded from above by $\left(\begin{array}{c}n-2 \\ 2\end{array}\right)$.

Proof. (1) Do an inflation of each mismatched region. This produces as many holes as mismatched regions, say, $m$ of them. Each hole is bounded by two triangulations of a polygon. This is guaranteed by the previous lemma. Denote by $k_{i}$ the number of vertices of the polygon associated with the $i$ th mismatched region. In each of the holes introduce an auxiliary interior point. The point can be used to triangulate the interior of the holes by filling in the holes with the coning of the vertex with the triangles it sees. We now have a triangulated ball.

Denote by $|D|$ the size of the original dissection. The triangulated ball has then $|D|+\sum_{i=1}^{m} 2\left(k_{i}-2\right)$ tetrahedra in total. The number of interior edges of this triangulation is the number of interior edges in the dissection, denoted by $e_{i}(D)$, plus the new additions; for each hole of length $k_{i}$ we added $k_{i}$ interior edges. In a triangulation $T$ of a 3-ball with $n$ boundary vertices and $n^{\prime}$ interior vertices, the number of tetrahedra $|T|$ is related to the number of interior edges $e_{i}$ of $T$ by the formula $|T|=n+e_{i}-n^{\prime}-3$. The proof is a simple application of Euler's formula for triangulated 2-spheres and 3 -balls and we omit the easy details.

Thus, we have the following equation:

$$
|D|+\sum_{i=1}^{m} 2\left(k_{i}-2\right)=n+e_{i}(D)+\sum_{i=1}^{m} k_{i}-m-3 .
$$

This can be rewritten as $|D|=n+e_{i}(D)-\sum_{i=1}^{m} k_{i}+3 m-3$. Taking into account that $e_{i}(D) \geq \sum_{i=1}^{m} 2\left(k_{i}-3\right)$ (because diagonals in a polygon are interior edges of the dissection), we get an inequality

$$
|D| \geq n+\sum_{i=1}^{m} k_{i}-3 m-3 .
$$

Finally, note that in a mismatching dissection we have $m \geq 1$ and $k_{i} \geq 4$. This gives the desired lower bound.

(2) Now we look at the proof of the upper bound on dissections. Given a 3dissection, we add tetrahedra of volume zero to complete a triangulation with flat simplices that has the same number of vertices. One can also think we are filling in the holes created by an inflation with (deformed) tetrahedra.

Lemma 3.1 states that mismatched regions were of the shape of convex polygons. The 2-simplices forming a mismatched region were divided into two groups (those becoming apart by an inflation). The two groups formed different triangulations of a convex polygon, and they had no interior edges in common. In this situation, we can make a sequence of flips (see [17]) between the two triangulations with the property that any edge once disappeared does not appear again (see Figure 8). We add one abstract, volume zero tetrahedron for each flip and obtain an abstract triangulation of a 3-ball.

The triangulation with flat simplices we created is a triangulated 3-ball with $n$ vertices. By adding a new point in a fourth dimension, and coning from the boundary 


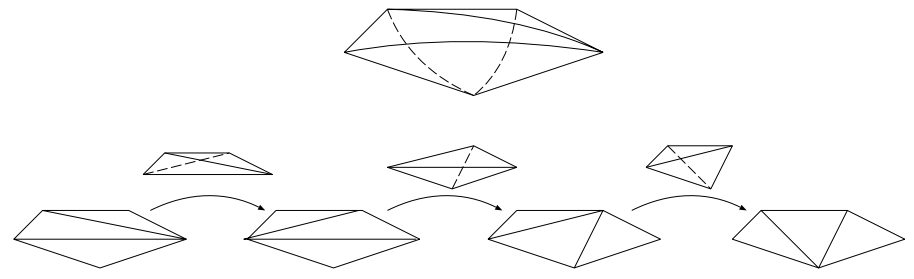

FIG. 8. Filling in holes with tetrahedra according to flips.

2-simplices to the point, we obtain a triangulated 3-sphere containing the original 3ball in its boundary. From the upper bound theorem for spheres (for an introduction to this topic, see [30]) its size is bounded from above by the number of facets of a cyclic 4 -polytope minus $2 n-4$, the number of 2 -simplices in the boundary of $D$. The fourdimensional cyclic polytope with $n+1$ vertices is well known to have $(n+1)(n-2) / 2$ facets (see [11, p. 63]), which completes the proof after a trivial algebraic calculation.

Open Problem 3.3. What is the correct upper bound theorem for dissections of $d$-dimensional polytopes with $d \geq 4$ ?

In our proof of Proposition 3.2 we built a triangulated PL-ball from a threedimensional dissection using the flip connectivity of triangulations of a convex $n$ gon. Unfortunately the same cannot be applied in higher dimensions, as the flip connectivity of triangulations of $d$-polytopes is known to be false for convex polytopes in general [22]. Even worse, however, the easy property we used from Lemma 3.1 that mismatched regions are convex polyhedra fails in dimension $d \geq 4$.

Proposition 3.4. The mismatched regions of a dissection of a convex 4-polytope can be nonconvex polyhedra.

Proof. The key idea is as follows. Suppose we have a 3-dimensional convex polytope $P$ and two triangulations $T_{1}$ and $T_{2}$ of it with the following properties: removing from $P$ the tetrahedra that $T_{1}$ and $T_{2}$ have in common, the rest is a nonconvex polyhedron $P^{\prime}$ such that the triangulations $T_{1}^{\prime}$ and $T_{2}^{\prime}$ of it obtained from $T_{1}$ and $T_{2}$ do not have any interior 2-simplex in common (actually, something weaker would suffice: that their common interior triangles, if any, do not divide the interior of the polytope).

In these conditions, we can construct the dissection we want as a bipyramid over $P$, coning $T_{1}$ to one of the apices and $T_{2}$ to the other one. The bipyramid over the nonconvex polyhedron $P^{\prime}$ will be a mismatched region of the dissection.

For a concrete example, start with Schönhardt's polyhedron whose vertices are labeled $1,2,3$ in the lower face and 4,5,6 in the top face. This is a nonconvex polyhedron made, for example, by twisting the three vertices on the top of a triangular prism. Add two antipodal points 7 and 8 close to the "top" triangular facets (those not breaking the quadrilaterals); see Figure 9. For example, take as coordinates for the points $1=(10,0,0), 2=(-6,8,0), 3=(-6,-8,0), 4=(10,-0.1,10), 5=(-6.1,8,10)$, $6=(-5.9,-8.1,10), 7=(0,0,10.1), 8=(0,0,-0.1)$.

Let $P^{\prime}$ be this nonconvex polyhedron and let $T_{1}^{\prime}=\{1278,1378,2378,1247,2457$, $2357,3567,1367,1467\}$ and $T_{2}^{\prime}=\{4578,4678,5678,1248,2458,2358,3568,1368$, $1468\}$. $T_{1}^{\prime}$ cones vertex 7 to the rest of the boundary of $P^{\prime}$, and $T_{2}^{\prime}$ cones vertex 8 . Any common interior triangle of $T_{1}^{\prime}$ and $T_{2}^{\prime}$ would use the edge 78 . But the link of 78 in $T_{1}^{\prime}$ contains only the points 1,2 , and 3 , and the link in $T_{2}^{\prime}$ contains only 4,5 , and 6 .

Let $P$ be the convex hull of the eight points, and let $T_{1}$ and $T_{2}$ be obtained from 

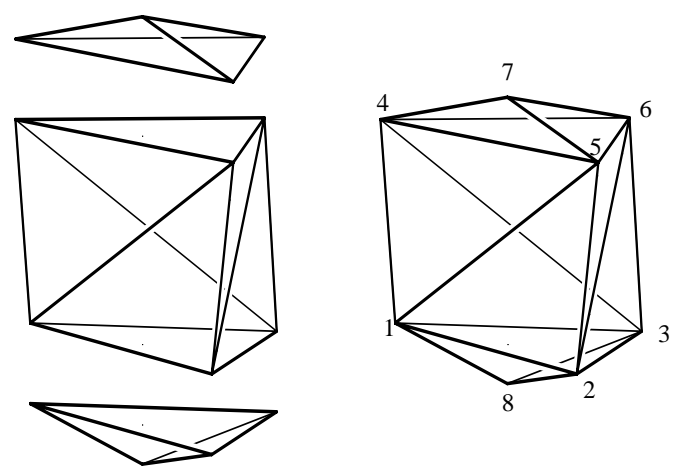

FIG. 9. The mismatched region of a four-dimensional dissection.

$T_{1}^{\prime}$ and $T_{2}^{\prime}$ by adding the three tetrahedra 1245,2356 , and 1346 .

4. Optimal dissections for specific polytopes. The regular cube has been widely studied for its smallest dissections $[12,14,18]$. This receives the name of simplexity of the cube. In contrast, because of the type of simplices inside a regular $d$ cube, a simple volume argument shows that the maximal size of a dissection is $d$ !, the same as for triangulations. On the other hand, we know that the size of the maximal triangulation of a combinatorial cube can be larger than that: For example, the combinatorial 3-cube obtained as the prism over a trapezoid (vertices on a parabola for instance) has triangulations of size 7 . Figure 10 shows a triangulation with seven simplices for those coordinatizations, where the edges $A B$ and $G H$ are not coplanar. The tetrahedron $A B G H$ splits the polytope into two nonconvex parts, each of which can be triangulated with three simplices. To see this, suppose that our polytope is a very small perturbation of a regular 3 -cube. In the regular cube, $A B G H$ becomes a diagonal plane which divides the cube into two triangular prisms, $A B C D G H$ and $A B E F G H$. In the nonregular cube, the diagonals $A H$ and $B G$, respectively, become nonconvex. Any pair of triangulations of the two prisms, each using the corresponding diagonal, together with tetrahedron $A B G H$ give a triangulation of the perturbed cube with seven tetrahedra. The boundary triangulation is shown in the flat diagram. It is worth noticing that for the regular cube the boundary triangulation we showed does not extend to a triangulation of the interior.

One can then ask, "What is the general growth for the size of a maximal dissection of a combinatorial cube?" To answer this question, at least partially, we use the above construction and we adapt an idea of Haiman, originally devised to produce small triangulations of regular cubes [12]. The idea is that from triangulations of a $d_{1}$ cube and a $d_{2}$-cube of sizes $s_{1}$ and $s_{2}$, respectively, we can get triangulations of the $\left(d_{1}+d_{2}\right)$-cube by first subdividing it into $s_{1} \times s_{2}$ copies of the product of two simplices of dimensions $d_{1}$ and $d_{2}$ and then triangulating each such piece. We recall that any triangulation of the Cartesian product of a $d_{1}$-simplex and a $d_{2}$-simplex has $\left(\begin{array}{c}d_{1}+d_{2} \\ d_{1}\end{array}\right)$ maximal simplices. Hence in total we have a triangulation of the $\left(d_{1}+d_{2}\right)$-cube into $s_{1} \times s_{2} \times\left(\begin{array}{c}d_{1}+d_{2} \\ d_{1}\end{array}\right)$ maximal simplices. Recursively, if one starts with a triangulation of size $s$ of the $d$-cube, one obtains triangulations for the $r d$-cube of size $(r d) !\left(\frac{s}{d !}\right)^{r}$. In Haiman's context one wants $s$ to be small, but here we want it to be big.

More precisely, denote by $f(d)$ the function $\max _{C}: d$-cube $\left(\max _{T}\right.$ of $\left.C|T|\right)$ and call 

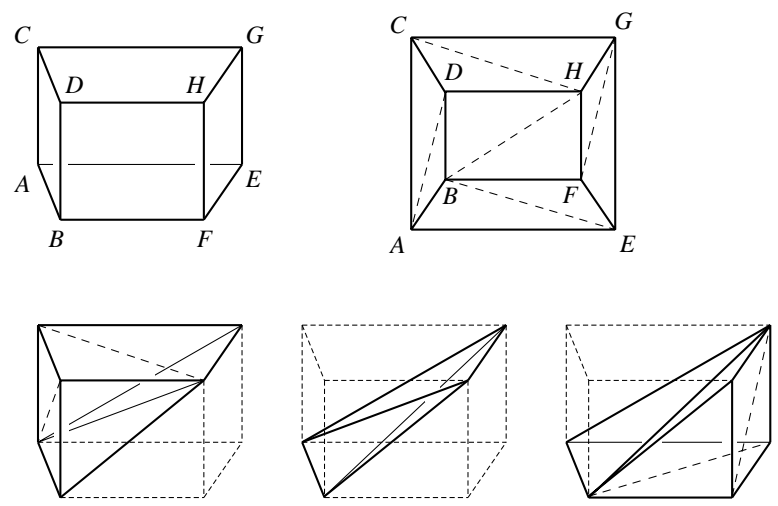

FIG. 10. A triangulation of a combinatorial 3-cube into seven tetrahedra.

$g(d)=(f(d) / d !)^{1 / d}$. Haiman's argument shows that if $f\left(d_{1}\right) \geq c_{1}{ }^{d_{1}} d_{1} !$ and $f\left(d_{2}\right) \geq$ $c_{2}{ }^{d_{2}} d_{2}$ ! for certain constants $c_{1}$ and $c_{2}$, then $f\left(d_{1}+d_{2}\right) \geq c_{1}{ }^{d_{1}} c_{2}{ }^{d_{2}}\left(d_{1}+d_{2}\right)$ ! (put differently, that $\left.g\left(d_{1}+d_{2}\right) \geq\left(g\left(d_{1}\right)^{d_{1}} g\left(d_{2}\right)^{d_{2}}\right)^{1 /\left(d_{1}+d_{2}\right)}\right)$. The value on the right-hand side is the weighted geometric mean of $g\left(d_{1}\right)$ and $g\left(d_{2}\right)$. In particular, if both $g\left(d_{1}\right)$ and $g\left(d_{2}\right)$ are $\geq 1$ and one of them is $>1$, then $g\left(d_{1}+d_{2}\right)$ is $>1$ as well.

We have constructed above a triangulation of size 7 for the Klee-Minty 3-cube, which proves $g(3) \geq \sqrt[3]{7 / 6}=1.053$. With Haiman's idea we can now construct "large" triangulations of certain 4-cubes and 5-cubes, which prove, respectively, that $g(4) \geq \sqrt[4]{7 / 6}=1.039$ and $g(5) \geq \sqrt[5]{7 / 6}=1.031$ (take $d_{1}=3$ and $d_{2}$ equal to one and two, respectively). Finally, since any $d>5$ can be expressed as a sum of 3's and 4's, we have $g(d) \geq \min \{g(3), g(4)\} \geq 1.039$ for any $d>5$. Hence we have the following proposition.

Proposition 4.1. For the family of combinatorial d-cubes with $d>2$ the function $f(d)=\max _{C \text { : } d \text {-cube }}\left(\max _{T}\right.$ of $\left.C|T|\right)$ admits the lower bound $f(d) \geq c^{d} d$ ! where $c \geq 1.031$.

Exactly as in Haiman's paper, the constant $c$ can be improved (asymptotically) if one starts with larger triangulations for the smaller-dimensional cubes. Using computer calculations (see Remark 4.5), we obtained a maximal triangulation for the Klee-Minty 4-cube with 38 maximal simplices, which shows that $g(d) \geq \sqrt[4]{38 / 24}=$ 1.122 for every $d$ divisible by 4 (see [1] for a complete study of this family of cubes). We omit listing the triangulation here, but it is available from the authors by request.

Open Problem 4.2. Is the sequence $g(d)$ bounded? In other words, is there an upper bound of type $c^{d} d$ ! for the function $f(d)$ ? Observe that the same question for minimal triangulations of the regular $d$-cube (whether there is a lower bound of type $c^{d} d$ ! for some $\left.c>0\right)$ is open as well. See [26] for the best lower bound known.

We continue our discussion with the study of optimal triangulations for threedimensional prisms and antiprisms. We will call an m-prism any 3-polytope with the combinatorial type of the product of a convex $m$-gon with a line segment. An $m$ antiprism will be any 3-polytope whose faces are two convex $m$-gons and $2 m$ triangles, each $m$-gon being adjacent to half of the triangles. Vertices of the two $m$-gons are connected with a band of alternately up and down pointing triangles.

Each such polyhedron has a regular coordinatization in which all the faces are regular polygons and a realization space which is the set of all possible coordinatizations that yield the same combinatorial information [20]. Our first result is valid in 
the whole realization space.

Proposition 4.3. For any three-dimensional m-prism, in any of its possible coordinatizations, the number of tetrahedra in a minimal triangulation is $2 m-5+\left\lceil\frac{m}{2}\right\rceil$.

For any three-dimensional $m$-antiprism, in any of its possible coordinatizations, the number of tetrahedra in a minimal triangulation is $3 m-5$.

Proof. In what follows we use the word cap to refer to the $m$-gon facets appearing in a prism or antiprism. We begin our discussion proving that any triangulation of the prism or antiprism has at least the size we state, and then we will construct triangulations with exactly that size.

We first prove that every triangulation of the $m$-prism requires at least $2 m-5+$ $\left\lceil\frac{m}{2}\right\rceil$ tetrahedra. We call a tetrahedron of the $m$-prism mixed if it has two vertices on the top cap and two vertices on the bottom cap of the prism; otherwise we say that the tetrahedron is top-supported when it has three vertices on the top (respectively, bottom-supported). For example, Figure 11 shows a triangulation of the regular 12prism in three slices. Parts (a) and (c) represent, respectively, the bottom and top caps. Part (b) is the intersection of the prism with the parallel plane at equal distance to both caps. In this intermediate slice, bottom or top supported tetrahedra appear as triangles, while mixed tetrahedra appear as quadrilaterals.

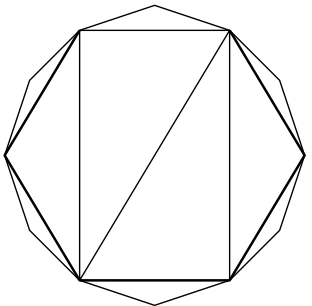

(a)

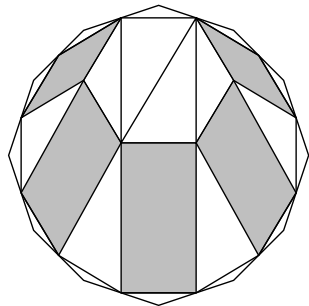

(b)

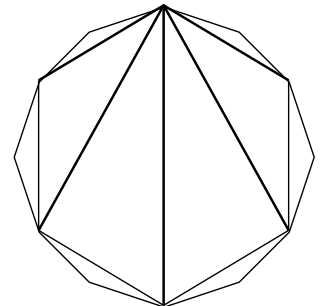

(c)

FiG. 11. A minimal triangulation of the regular 12-prism.

Because all triangulations of an $m$-gon have $m-2$ triangles there are always exactly $2 m-4$ tetrahedra that are bottom- or top-supported. In the rest, we show there are at least $\left\lceil\frac{m}{2}\right\rceil-1$ mixed tetrahedra. Each mixed tetrahedra marks an edge of the top, namely, the edge it uses from the top cap. Of course, several mixed tetrahedra could mark the same top edge. Group together top-supported tetrahedra that have the same bottom vertex. This grouping breaks the triangulated top $m$-gon into polygonal regions. Note that every edge between two of these regions must be marked. For example, in part (c) of Figure 11 the top cap is divided into six regions by five marked edges (the thick edges in the figure). Let $r$ equal the number of regions under the equivalence relation we set. There are $r-1$ interior edges separating the $r$ regions, and all of them are marked. Some boundary edges of the top cap may be marked too (none of them is marked in the example of Figure 11).

We can estimate the marked edges in another way: There are $m$ edges on the boundary of the top, which appear partitioned among some of the regions (it could be the case some region does not contain any boundary edge of the $m$-gon). We claim that no more than two boundary edges per region will be unmarked $(*)$. This follows because a boundary edge is not marked only when the top-supported tetrahedron that contains it has the point in the bottom cap that is directly under one of the vertices of the edge. In a region, at most two boundary edges can satisfy this. Hence we get 
at least $m-2 r$ marked edges on the boundary of the top and at least $(r-1)+(m-$ $2 r)=m-r-1$ marked edges in total. Thus the number of mixed tetrahedra is at least the maximum of $r-1$ and $m-r-1$. In conclusion, we get that, indeed, the number of mixed tetrahedra is bounded below by $\left\lceil\frac{m}{2}\right\rceil-1$. Note that we use only the combinatorics and convexity of the prism in our arguments. We will show that minimal triangulations achieve this lower bound but then observe that if $m$ is even, in a minimal triangulation we must have $r=m / 2$ and no boundary edge can be marked, as is the case in Figure 11. If $m$ is odd, then we must have $r \in\{(m-1) / 2,(m+1) / 2\}$ and at most one boundary edge can be marked.

The proof that any triangulation of an $m$-antiprism includes at least $3 m-5$ tetrahedra is similar. There are $2 m-4$ top-supported and bottom-supported tetrahedra in any triangulation and there are $r-1$ marked edges between the regions in the top. The only difference is that, instead of claim $(*)$, one has at most one unmarked boundary edge per region. Thus there are at least $m-r$ marked edges in the boundary of the top and in total at least $(r-1)+(m-r)=m-1$ marked edges in the top. Hence there exist at least $(2 m-4)+(m-1)=3 m-5$ tetrahedra in any triangulation.

For an $m$-antiprism we can easily create a triangulation of size $3 m-5$ by choosing any triangulation of the bottom $m$-gon and then coning a chosen vertex $v$ of the top $m$-gon to the $m-2$ triangles in that triangulation and to the $2 m-3$ triangular facets of the $m$-antiprism which do not contain $v$. This construction is exhibited in Figure 12. Parts (a) and (c) show the bottom and top caps triangulated (each with its five marked edges) and part (b) shows an intermediate slice with the five mixed tetrahedra appearing as quadrilaterals.

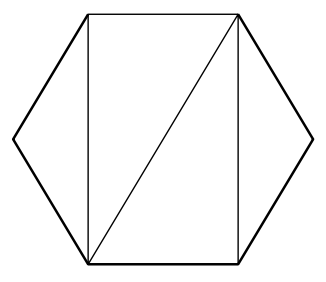

(a)

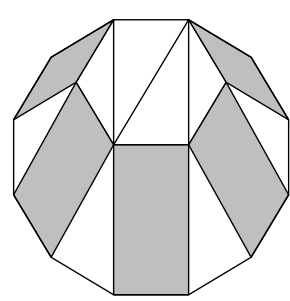

(b)

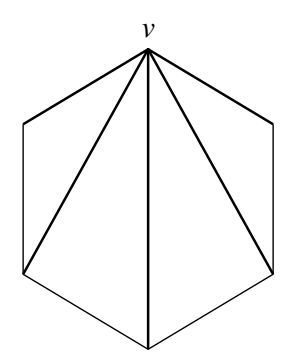

(c)

FIG. 12. A minimal triangulation of the regular 6-antiprism.

For an $m$-prism, let $u_{i}$ and $v_{i}, i=1, \ldots, m$ denote the top and bottom vertices, respectively, so that the vertices of each cap are labeled consecutively and $u_{i} v_{i}$ is always an edge of the prism.

If $m$ is even we can chop off the vertices $u_{i}$ for odd $i$ and $v_{j}$ for even $j$, so that the prism is decomposed into $m$ tetrahedra and an $\left(\frac{m}{2}\right)$-antiprism. The antiprism can be triangulated into $\frac{3 m}{2}-5$ tetrahedra, which gives a triangulation of the prism into $\frac{5 m}{2}-5$ tetrahedra, as desired. Actually, this is how the triangulation of Figure 11 can be obtained from that of Figure 12.

If $m$ is odd we do the same, except that we chop off only the vertices $u_{1}, \ldots, u_{m-2}$ and $v_{2}, \ldots, v_{m-1}$ (no vertex is chopped in the edge $u_{m} v_{m}$ ). This produces $m-$ 1 tetrahedra and an $\left(\frac{m+1}{2}\right)$-antiprism. We triangulate the antiprism into $\frac{3 m+3}{2}-$ 5 tetrahedra and this gives a triangulation of the $m$-prism into $\frac{5 m+1}{2}-5$ tetrahedra. 
We have seen that the coordinates are not important when calculating minimal triangulations of the three-dimensional prisms and antiprisms. On the other hand, the difference in size of the maximal triangulation can be quite dramatic. Below we prove that in certain coordinatizations it is roughly $\frac{m^{2}}{2}$ and show experimental data indicating that for the regular prism it is close to $\frac{m^{2}}{4}$.

Proposition 4.4. Let $A_{m}$ be a prism of order $m$ with all its side edges parallel.

(1) The size of a maximal triangulation of $A_{m}$ is bounded as

$$
\left\lceil\frac{m^{2}+6 m-16}{4}\right\rceil \leq \max _{T} \text { of } A_{m}|T| \leq \frac{m^{2}+m-6}{2} .
$$

(2) The upper bound is achieved if the two caps (m-gon facets) are parallel and there is a direction in which the whole prism projects onto one of its side quadrangular facets. (For a concrete example, let one of the m-gon facets have vertices on a parabola and let $A_{m}$ be the product of it with a segment.)

Proof. Let the vertices of the prism be labeled $u_{1}, \ldots, u_{m}$ and $v_{1}, \ldots, v_{m}$ so that the $u_{i}$ 's and the $v_{j}$ 's form the two caps, vertices in each cap are labeled consecutively, and $u_{i} v_{i}$ is always a side edge.

For the upper bound in part (1), we have to prove that a triangulation of $A_{m}$ has at most $\frac{m^{2}+m-6}{2}-2 m+3=\frac{m(m-3)}{2}$ interior diagonals. The possible diagonals are the edges $u_{i} v_{j}$, where $i-j$ is not in $\{-1,0,1\}$ modulo $m$. This gives exactly twice the number we want. However, for any $i$ and $j$ the diagonals $u_{i} v_{j}$ and $u_{j} v_{i}$ intersect, so only one of them can appear in each triangulation.

We now prove that the upper bound is achieved if $A_{m}$ is in the conditions of part (2). In fact, the condition on $A_{m}$ that we will need is that for any $1 \leq i<j \leq k<l \leq$ $m$, the point $v_{j}$ sees the triangle $v_{i} u_{k} u_{l}$ from the same side as $v_{k}$ and $v_{l}$ (i.e., "from above" if we call top cap the one containing the $v_{i}$ 's). With this we can construct a triangulation with $\frac{m^{2}+m-6}{2}=\left(\begin{array}{c}m-1 \\ 2\end{array}\right)+2 m-4$ tetrahedra as follows.

First cone the vertex $v_{1}$ to any triangulation of the bottom cap (this gives $m-2$ tetrahedra). The $m-2$ upper boundary facets of this cone are visible from $v_{2}$, and we cone them to it (again $m-2$ tetrahedra). The new $m-2$ upper facets are visible from $v_{3}$ and we cone them to it ( $m-2$ tetrahedra more). Now one of the upper facets of the triangulation is $v_{1} v_{2} v_{3}$, part of the upper cap, but the other $m-3$ are visible from $v_{4}$, so we cone them and introduce $m-4$ tetrahedra. Continuing the process, we will introduce $m-4, m-5, \ldots, 2,1$ tetrahedra when coning the vertices $v_{5}, v_{6}, \ldots, v_{m-1}, v_{m}$, which gives a total of $\left(\begin{array}{c}m-1 \\ 2\end{array}\right)+2 m-4$ tetrahedra, as desired.

The triangulation we have constructed is the placing triangulation [17] associated with any ordering of the vertices finishing with $v_{1}, \ldots, v_{m}$. A different description of the same triangulation is that it cones the bottom cap to $v_{1}$, the top cap to $u_{m}$, and its mixed tetrahedra are all the possible $v_{i} v_{i+1} u_{j} u_{j+1}$ for $1 \leq i<j \leq m-1$. This gives $\left(\begin{array}{c}m-1 \\ 2\end{array}\right)$ mixed tetrahedra and $\left(\begin{array}{c}m-1 \\ 2\end{array}\right)+2 m-4$ tetrahedra in total.

We finally prove the lower bound stated in part (1). Without loss of generality, we can assume that our prism has its two caps parallel (if not, do a projective transformation keeping the side edges parallel). Then, $A_{m}$ can be divided into two prisms in the conditions of part (2) of sizes $k$ and $l$ with $k+l=m+2$ : take any two side edges of $A_{m}$ which possess parallel supporting planes and cut $A_{m}$ along the plane containing both edges. By part (2), we can triangulate the two subprisms with $\left(\begin{array}{c}k+1 \\ 2\end{array}\right)-3$ and $\left(\begin{array}{c}l+1 \\ 2\end{array}\right)-3$ tetrahedra, respectively, taking care that the two triangulations use the same diagonal in the dividing plane. This gives a triangulation of $A_{m}$ with 
$\left(\begin{array}{c}k+1 \\ 2\end{array}\right)+\left(\begin{array}{c}l+1 \\ 2\end{array}\right)-6=\frac{k^{2}+l^{2}+m-10}{2}$ tetrahedra. This expression achieves its minimum when $k$ and $l$ are as similar as possible, i.e., $k=\left\lfloor\frac{m}{2}\right\rfloor+1$ and $l=\left\lceil\frac{m}{2}\right\rceil+1$. Plugging these values in the expression gives a triangulation of size $\left\lceil\frac{m^{2}+6 m-16}{4}\right\rceil$.

Based on an integer programming approach we can compute maximal triangulations of specific polytopes (see Remark 4.5). Our computations with regular prisms up to $m=12$ show that the size of their maximal triangulations achieve the lower bound stated in part (1) of Proposition 4.4 (see Table 2). In other words, they show that the procedure of dividing them into two prisms of sizes $\left\lfloor\frac{m}{2}\right\rfloor+1$ and $\left\lceil\frac{m}{2}\right\rceil+1$ in the conditions of part (2) of Proposition 4.4 and triangulating the subprisms independently yields maximal triangulations.

We have also computed maximal sizes of triangulations for the regular $m$-antiprisms up to $m=12$, which turn out to follow the formula $\left\lfloor\frac{m^{2}+8 m-16}{4}\right\rfloor$. A construction of a triangulation of this size for every $m$ can be made as follows: Let the vertices of the regular $m$-antiprism be labeled $u_{1}, \ldots, u_{m}$ and $v_{1}, \ldots, v_{m}$ so they are forming the vertices of the two caps consecutively in this order and $v_{i} u_{i}$ and $u_{i} v_{i+1}$ are side edges. We let $v_{m+1}=v_{1}$. The triangulation is made by placing the vertices in any ordering finishing with $v_{1}, v_{2}, v_{m}, v_{3}, v_{m-1}, \ldots, v_{\left\lceil\frac{m}{2}\right\rceil+1}$. The tetrahedra used are the bottom-supported tetrahedra with apex $v_{1}$, top-supported tetrahedra with apex $u_{\left\lceil\frac{m}{2}\right\rceil}$, and the mixed tetrahedra $v_{i} v_{i+1} u_{j} u_{j+1}$ for $1 \leq i \leq j \leq\left\lfloor\frac{m}{2}\right\rfloor$ and $u_{i} u_{i+1} v_{j} v_{j+1}$ for $\left\lfloor\frac{m}{2}\right\rfloor+1 \leq i<j \leq m$.

We conjecture that these formulas for regular base prisms and antiprisms actually give the sizes of their maximal triangulations for every $m$, but we do not have a proof.

TABLE 2

Sizes of maximal triangulations of prisms and antiprisms.

\begin{tabular}{|c|c|c|c|c|c|c|c|c|c|c|}
\hline$m$ & 3 & 4 & 5 & 6 & 7 & 8 & 9 & 10 & 11 & 12 \\
\hline Prism (regular base) & 3 & 6 & 10 & 14 & 19 & 24 & 30 & 36 & 43 & 50 \\
\hline Antiprism (regular base) & 4 & 8 & 12 & 17 & 22 & 28 & 34 & 41 & 48 & 56 \\
\hline
\end{tabular}

Remark 4.5. How can one find minimal and maximal triangulations in specific instances? The approach we followed for computing Tables 1 and 2 and some of the results in Proposition 2.3 is the one proposed in [8], based on the solution of an integer programming problem. We think of the triangulations of a polytope as the vertices of the following high-dimensional polytope: Let $A$ be a $d$-dimensional polytope with $n$ vertices. Let $N$ be the number of $d$-simplices in $A$. We define $P_{A}$ as the convex hull in $\boldsymbol{R}^{N}$ of the set of incidence vectors of all triangulations of $A$. For a triangulation $T$ the incidence vector $v_{T}$ has coordinates $\left(v_{T}\right)_{\sigma}=1$ if $\sigma \in T$ and $\left(v_{T}\right)_{\sigma}=0$ if $\sigma \notin T$. The polytope $P_{A}$ is the universal polytope defined in general by Billera, Filliman, and Sturmfels [3], although it appeared in the case of polygons in [7]. In [8], it was shown that the vertices of $P_{A}$ are precisely the integral points inside a polyhedron that has a simple description in terms of the oriented matroid of $A$ (see [8] for information on oriented matroids). The concrete integer programming problems were solved using $C$-plex Linear Solver ${ }^{T M}$. The program to generate the linear constraints is a small $C^{++}$program written by Samuel Peterson and the first author. Source code, brief instructions, and data files are available via ftp at http://www.math.ucdavis.edu/ deloera. An alternative implementation by Tajima is also available $[27,28]$. He used his program to corroborate some of these results.

It should be mentioned that a simple variation of the ideas in [8] provides enough 
equations for an integer program whose feasible vertices are precisely the $0 / 1$-vectors of dissections. The incidence vectors of dissections of $\operatorname{conv}(A)$, for a point set $A$, are just the $0 / 1$ solutions to the system of equations $\left\langle x, v_{T}\right\rangle=1$, where $v_{T}$ 's are the incidence vectors for every regular triangulation $T$ of the Gale transform $A^{*}$ (regular triangulations in the Gale transform are the same as chambers in $A$ ). Generating all these equations is as hard as enumerating all the chambers of $A$. Nevertheless, it is enough to use those equations coming from placing triangulations (see [23, section $32]$ ), which gives a total of about $n^{d+1}$ equations if $A$ has $n$ points and dimension $d$.

Acknowledgments. We are grateful to Alexander Below and Jürgen RichterGebert for their help and ideas in the proofs of Propositions 3.2 and 3.4. Alexander Below made Figure 9 using the package Cinderella. The authors thank Akira Tajima and Jörg Rambau for corroborating many of the computational results. We thank Samuel Peterson for his help with our calculations. Finally, we thank Hiroshi Imai, Bernd Sturmfels, and Akira Tajima for their support of this project.

\section{REFERENCES}

[1] N. Amenta and G.M. Ziegler, Deformed products and maximal shadows of polytopes, in Advances in Discrete and Computational Geometry, B. Chazelle, J.E. Goodman, and R. Pollack, eds., Contemp. Math. 223, AMS, Providence, RI, 1999, pp. 57-90.

[2] A. Barvinok and J. Pommersheim, An algorithmic theory of lattice points in polyhedra, in New Perspectives in Algebraic Combinatorics, Math. Sci. Res. Inst. Publ. 38, Cambridge University Press, Cambridge, UK, 1999.

[3] L. Billera, P. Filliman, and B. Sturmfels, Constructions and complexity of secondary polytopes, Adv. Math., 83 (1990), pp. 155-179.

[4] A. Below, U. Brehm, J.A. De Loera, and J. Richter-Gebert, Minimal simplicial dissections and triangulations of convex 3-polytopes, Discrete Comput. Geom., 24 (2000), pp. $35-48$.

[5] W. Bruns, J. Gubeladse, and N.V. Trung, Normal polytopes, triangulations and Koszul algebras J. Reine Angew. Math., 485 (1997), pp. 123-160.

[6] H.S.M. Coxeter, Regular Polytopes, Dover, New York, 1973.

[7] G.B. Dantzig, A.J. Hoffman, And T.C. Hu, Triangulations (tilings) and certain block triangular matrices, Math. Programming, 31 (1985), pp. 1-14.

[8] J.A. De Loera, S. Hoşten, F. Santos, And B. Sturmfels, The polytope of all triangulations of a point configuration, Doc. Math., 1 (1996), pp. 103-119.

[9] H. Edelsbrunner, F.P. Preparata, and D.B. West, Tetrahedrizing point sets in three dimensions, J. Symbolic Comput., 10 (1990), pp. 335-347.

[10] R.T. Firla AND G.M. Ziegler, Hilbert bases, unimodular triangulations, and binary covers of rational polyhedral cones, Discrete Comput. Geom., 21 (1999), pp. 205-216.

[11] B. Grünbaum, Convex Polytopes, Interscience, London, 1967.

[12] M. Haiman, A simple and relatively efficient triangulation of the $n$-cube, Discrete Comput. Geom., 6 (1991), pp. 287-289.

[13] B. Huber, J. Rambau, and F. Santos, The Cayley trick, lifting subdivisions and the BohneDress theorem on zonotopal tilings, J. Eur. Math. Soc. (JEMS), 2 (2000), pp. 179-198.

[14] R.B. Hughes and M.R. Anderson, Simplexity of the cube, Discrete Math., 158 (1996), pp. 99-150.

[15] J.-M. KANTOR, Triangulations of integral polytopes and Ehrhart polynomials, Beiträge Algebra Geom., 39 (1998), pp. 205-218.

[16] J. Lagarias and G.M. Ziegler, Unimodular Triangulations, manuscript, 1999.

[17] C.W. LEE, Subdivisions and triangulations of polytopes, in Handbook of Discrete and Computational Geometry, J.E. Goodman and J. O'Rourke, eds., CRC Press, Boca Raton, FL, 1997, pp. 271-290.

[18] P.S. Mara, Triangulations for the cube, J. Combin. Theory Ser. A, 20 (1976), pp. 170-177.

[19] J. Rambau, TOPCOM: A Program for Computing All Triangulations of a Point Set, ZIBBerlin, 1999; also available online from http://www.zib.de/rambau/TOPCOM.html.

[20] J. Richter-Gebert, Realization Spaces of Polytopes, Lecture Notes in Math. 1643, SpringerVerlag, Berlin, 1996. 
[21] G.L. Rothschild and E.G. Straus, On triangulations of the convex hull of $n$ points, Combinatorica, 5 (1985), pp. 167-179.

[22] F. Santos, A point configuration whose space of triangulations is disconnected, J. Amer. Math. Soc., 13 (2000), pp. 611-637.

[23] F. Santos, Triangulations of oriented matroids, Mem. Amer. Math. Soc., to appear; also available online from http://www.matesco.unican.es/ santos/Articulos/index.html.

[24] А. Sевӧ, Hilbert bases, Carathéodory's theorem and combinatorial optimization, in Integer Programming and Combinatorial Optimization, R. Kannan and W. Pulleyblank, eds., Math. Programming Society, University of Waterloo Press, Waterloo, Ontario, Canada, 1990, pp. 431-456.

[25] А. SєвÖ, An Introduction to Empty Lattice Simplices, manuscript; also available online from http://cosmos.imag.fr/DMD/OPTICOMB/Membres/sebo/sebo.html.

[26] W.D. Smith, A lower bound for the simplexity of the $n$-cube via hyperbolic volumes, European J. Combin., 21 (2000), pp. 131-137.

[27] A. TAJIMA, Optimality and integer programming formulations of triangulations in general dimension, in Proceedings of 9th Annual International Symposium on Algorithms and Computation (ISAAC '98), K.-Y. Chwa and O.H. Ibarra, eds., Lecture Notes in Comput. Sci. 1533, Springer-Verlag, Berlin, pp. 377-386.

[28] A. Tajima, Optimizing Geometric Triangulations by Using Integer Programming, Ph.D. thesis, University of Tokyo, Tokyo, Japan 2000; also available online from http://www-imai.is.s.utokyo.ac.jp/ akira/papers/dissertation.pdf.

[29] F. Takeuchi and H. Imai, Enumerating triangulations for products of two simplices and for arbitrary configurations of points, in Proceedings of 3rd Annual International Conference on Computing and Combinatorics (COCOON '97), T. Jiang and D. T. Lee, eds., Lecture Notes in Comput. Sci. 1276, Springer-Verlag, Berlin, pp. 470-481.

[30] G.M. Ziegler, Lectures on Polytopes, Springer-Verlag, New York, 1995. 